\title{
Strigolactones in the Rhizosphere: Friend or Foe?
}

\author{
Carolien De Cuyper and Sofie Goormachtig ${ }^{\dagger}$ \\ Department of Plant Biotechnology and Bioinformatics, Ghent University, and Center for Plant Systems Biology, VIB, 9052 \\ Ghent, Belgium
}

Accepted 8 June 2017.

\begin{abstract}
Strigolactones are well-known endogenous plant hormones that play a major role in planta by influencing different physiological processes. Moreover, ex planta, strigolactones are important signaling molecules in root exudates and function as host detection cues to launch mutualistic interactions with arbuscular mycorrhizal fungi in the rhizosphere. However, parasitic plants belonging to the Orobanchaceae family hijacked this communication system to stimulate their seed germination when in close proximity to the roots of a suitable host. As a result, the secretion of strigolactones by the plant can have both favorable and detrimental outcomes. Here, we discuss these dual positive and negative effects of strigolactones and we provide a detailed overview on the role of these molecules in the complex dialogs between plants and different organisms in the rhizosphere.
\end{abstract}

\section{Strigolactone, a versatile molecule.}

Belowground as well as aboveground, plants encounter various organisms (Buée et al. 2009). In the soil, these interactions take place in the rhizosphere, the narrow zone of soil surrounding the root in which different microorganisms reside, including neutral, beneficial, and harmful ones (Raaijmakers et al. 2009). Plants need to be able to cope with all of them; they should defend themselves against pathogens, while simultaneously trying to establish mutualistic associations. To accomplish this challenging task, plant roots release a wide range of communication molecules in the rhizosphere, e.g., polysaccharides, amino acids, aliphatic acids, aromatic acids, fatty acids, sterols, phenols, and other secondary metabolites, including strigolactones (Bais et al. 2006; Steinkellner et al. 2007; Venturi and Keel 2016).

Strigolactones are present in root exudates of diverse monocotyledonous and dicotyledonous plant species at very low concentrations (picomolar and nanomolar) (Xie 2016). In the soil, these molecules are implicated in the communication between plants and a wide range of parasites belonging to the Orobanchaceae family (Cook et al. 1966; Yoneyama et al. 2010) but, also, in the communication with arbuscular mycorrhizal fungi (Akiyama et al. 2005) and, more recently, in the legume-rhizobia symbiosis (De Cuyper et al. 2015; Foo and Davies 2011; PeláezVico et al. 2016; Soto et al. 2010). In addition to their importance as rhizosphere molecules, strigolactones are also studied for their role as plant hormones that influence various physiological processes to adjust plant growth and development, such as shoot branching, secondary growth processes, leaf shape and senescence,

${ }^{\dagger}$ Corresponding author: S. Goormachtig;

E-mail: sofie.goormachtig@psb.vib-ugent.be

(c) 2017 The American Phytopathological Society internode elongation, and environmental stress responses (Al-Babili and Bouwmeester 2015).

The presence of strigolactones in algae indicates that these molecules appeared in evolution before the first arbuscular mycorrhizal symbiosis, suggesting that their original function was to act as plant hormones that later evolved into rhizosphere communication signals (Delaux et al. 2012). Indeed, strigolactones stimulate rhizoid elongation of Charales, liverworts, and mosses, thereby increasing their anchorage ability, an essential trait for terrestrial colonization 460 million years ago (Delaux et al. 2012). By their action, both as phytohormones in planta and as rhizosphere signaling molecules ex planta, strigolactones allow plants to adapt flexibly to different biotic or abiotic stress conditions by establishing mutualistic interactions, by playing a potential role in plant-pathogen interactions, and, by coordinating root and shoot architecture. Here, we give a detailed overview on the role of strigolactones in the complex dialogs between plants and other rhizosphere members.

From synthesis to secretion.

Strigolactones are synthesized from carotenoids through the consecutive action of DWARF 27 (D27), CAROTENOID CLEAVAGE DIOXYGENASE 7 (CCD7), CCD8, and MORE AXILLARY GROWTH 1 (MAX1). In the plastids, the $\beta$-carotene isomerase D27 and carotenoid cleavage dioxygenases CCD7 and CCD8 convert the strigolactone precursor all-trans- $\beta$-carotene into the strigolactone biosynthetic precursor, carlactone (Alder et al. 2012; Booker et al. 2004; Bruno et al. 2017; Kohlen et al. 2012; Lin et al. 2009; Seto et al. 2014). In the cytosol, the cytochrome P450 enzyme MAX1 catalyzes carlactone oxidations to produce carlactonoic acid, which is further methylated and, subsequently, is oxidized by LATERAL BRANCHING OXIDOREDUCTASE, an oxidoreductase-like enzyme of the 2-oxoglutarate and Fe (II)dependent dioxygenase superfamily, into biologically active strigolactone-like compounds (Alder et al. 2012; Booker et al. 2005; Brewer et al. 2016; Kohlen et al. 2011; Seto et al. 2014; Zhang et al. 2014). Despite our understanding of strigolactone biosynthesis, it remains unclear whether canonical strigolactones or rather carlactone-derived molecules are synthesized in Arabidopsis thaliana and it is still unknown which ones act as endogenous hormones (Abe et al. 2014; Kohlen et al. 2011).

Today, at least 20 different natural canonical strigolactones have been structurally characterized, all consisting of four rings, designated $\mathrm{A}$ to $\mathrm{D}$ rings (Tokunaga et al. 2015). The A, B, and $\mathrm{C}$ rings carry a lactone group to which the $\mathrm{D}$ ring is connected via an enol ether bridge. The C-D ring is highly conserved among the different strigolactones and is essential for its bioactivity, whereas the A and B rings can have various modifications (Yoneyama et al. 2013; Zwanenburg and Pospísil 2013). Different plant species produce different strigolactone 
blends. Structure-activity relationship (SAR) studies have indicated that the chemical characteristics might be correlated with specific outcomes; for instance, strigolactones with a hydroxyl group have the highest activity in parasitic weed seed germination (Ćavar et al. 2015). Understanding the specificity of these structural modifications allows the selection of plant varieties with a favorable strigolactone 'fingerprint' that is not recognized by parasitic seeds but still promotes mutualistic interactions. Instead of natural strigolactones, the synthetic strigolactone rac-GR24 is widely used in strigolactone research. This racemic mixture consists of both 2'R-configured molecules that mimic the configuration of the natural strigolactones and the 2' $L$ configurations that are not found in nature. Nevertheless, as discussed below in more detail, these noncanonical forms are perceived by plants, not via the strigolactone, but via the karrkinin signaling pathway (Scaffidi et al. 2014). Hence, studies with rac-GR24 should always be interpreted within this context.

Different expression studies and grafting experiments have revealed that strigolactones might not exclusively be produced in the root but might be more generally synthesized in the vascular tissues of many organs (Borghi et al. 2016). In contrast, a long-distance transport from the root to the shoot has been considered as well. Indeed, the discovery of both strigolactones and carlactone in the xylem sap of Arabidopsis and Solanum lycopersicum (tomato) hinted at a root-derived strigolactone trafficking to the shoot via the xylem (Kohlen et al. 2011, 2012). However, in another study, no strigolactones were detected in the xylem sap of various species, although a slow root-to-shoot transport was still observed, suggesting an active cell-to-cell transport instead of a fast movement via the xylem sap stream (Xie et al. 2015b).

Knowledge about the strigolactone secretion from the root into the soil is essential to fully understand the role played by strigolactones in rhizosphere communication. Thus far, the only well-characterized strigolactone transporter is PLEIOTROPIC DRUG RESISTANCE 1 (PDR1) in Petunia hybrida (petunia) (Kretzschmar et al. 2012), belonging to the G-type subfamily of ATP-BINDING CASSETTE (ABC) transporters, known to be involved in the directional transport of several phytohormones, such as cytokinin, abscisic acid, and auxin (Borghi et al. 2015). Mutants are characterized by an enhanced branching phenotype and their root exudates induce less efficiently arbuscular mycorrhizal hyphal branching and parasitic seed germination. Moreover, Arabidopsis plants overexpressing PDRl of Petunia axillaris secreted larger amounts of the synthetic strigolactone analog rac-GR24 than the wild-type plants. Taken together, these findings support a role for PDR1 as a strigolactone exporter that mediates strigolactone transport in the plant as well as into the soil (Fig. 1). The asymmetrical localization of PDR1 at the plasma membrane is the reason for the strigolactone export out of the cell. Indeed, in the root tip, PDR1 is localized at the apical membrane of root hypodermal cells, indicative for an active strigolactone transport from the root tip to the shoot. In contrast, above the root tip, PDR1 is located at the outer lateral membrane of hypodermal passage cells, known to be specific entry sites for arbuscular mycorrhizal fungi that enable an active outward traffic into the rhizosphere (Sasse et al. 2015; Sharda and Koide 2008). In addition to the root, PDRl is also expressed in the stem nodes, close to the axillary buds. Interestingly, because the PDRI expression is regulated by different factors, such as colonization of arbuscular mycorrhizal fungi, treatment with rac-GR24 or auxin, and nutrient conditions (mainly phosphate) in the soil, this strigolactone exporter might synchronize plant growth with nutrient availability (Kretzschmar et al. 2012). In addition to petunia, in Nicotiana tabacum (tobacco), a close PDR1 homolog, PDR6, has been identified that could be involved in strigolactone transport (Xie et al. 2015a). However, in Arabidopsis, the closest PDRI homolog, AtABCG40, has been described as an abscisic acid transporter, demonstrating that sequence information might not always correlate with the transporter's substrate (Borghi et al. 2016; Kang et al. 2010).

Strigolactones are inherently unstable in the soil, due to the labile ether bond that spontaneously hydrolyzes in water environments and inactivates these molecules (Akiyama et al. 2010). The hydrolysis rate strongly depends on strigolactone structure and environmental conditions. For instance, under neutral conditions, the half-life of the synthetic strigolactone analog rac-GR24 was estimated to be 10 days (Akiyama et al. 2010), but natural strigolactones are much more unstable than synthetic ones; for instance, the half-life of 5-deoxystrigol has been reported to be only 1.5 days (Akiyama et al. 2010). Because strigolactones are short-living compounds, partners can only react to these fragile molecules in close vicinity of the root, i.e., in the rhizosphere. Furthermore, a concentration gradient is expected to be formed that can be used by different organisms as an indicator for the distance to their host.

\section{A cue for arbuscular mycorrhizal and other fungi.}

Originally, strigolactones were discovered as seed germination stimulants of parasitic weeds (Cook et al. 1966). Immediately, the question arose why plants would release molecules that induce germination of their parasites. Therefore, strigolactones have been suggested to possess other functions that outweigh the negative effects of parasitism, resulting in a selective pressure to maintain strigolactone biosynthesis in nature. Indeed, a beneficial role for strigolactones has been unveiled, more particularly, in enhancing arbuscular mycorrhization, which is an important fungal symbiosis with roots of approximately $80 \%$ of the vascular plants (Akiyama et al. 2005). Arbuscular mycorrhizal fungi belong to the phylum Glomeromycota and are obligate symbionts that rely on their hosts to complete their development. They enhance uptake by the plant of water and mineral nutrients, especially inorganic phosphate $(\mathrm{Pi})$ and, in return, they receive carbohydrates from their host. Molecular data and fossil studies suggest that this symbiosis evolved 400 million years ago and is of major importance in the colonization of land by plants (Remy et al. 1994). Hence, this symbiosis is assumed to exist much longer than the association with parasitic plants. In other words, the primary role of strigolactones in rhizosphere communication has been to stimulate the interaction with arbuscular mycorrhizal fungi and, later on, parasitic plants have hijacked this system to detect their hosts (Leyser 2008).

Upon host recognition, one of the first responses of arbuscular mycorrhizal fungi involves the extensive branching of hyphae to ensure a good contact with the host root, necessary for the further establishment of symbiosis (Giovannetti et al. 1993, 1994). Host roots release signal molecules, referred to as branching factors, that stimulate hyphal branching of several arbuscular mycorrhizal fungi species (Buee et al. 2000; Giovannetti et al. 1996). This branching factor has been isolated and identified as 5-deoxystrigol (Akiyama et al. 2005). Other strigolactones have been reported to induce hyphal branching as well (Akiyama et al. 2005; Mori et al. 2016), thereby forming a large network of mycelia that reaches out far beyond the root rhizosphere, allowing the capture of nutrients from expanded soil areas. In agreement, the colonization rates of the arbuscular mycorrhizal fungi Glomus intraradices and Gigaspora rosea have been found to be reduced in strigolactone-deficient tomato and Zea mays (maize) mutants, respectively (Gomez-Roldan et al. 2007; Koltai et al. 2010). In addition to hyphal branching, rac-GR24 also 
stimulates spore germination and boosts fungal metabolism (Besserer et al. 2006, 2008).

As arbuscular mycorrhizal fungi respond to strigolactones, they must be able to sense them. How this perception is established is still unknown. Inside plants, strigolactones are recognized by the $\alpha / \beta$ hydrolase DWARF 14 (D14), after which a signaling complex is made, consisting of the Skp1-Cullin-F-box (SCF) complex with the F-box protein MORE AXILLARY GROWTH 2 (MAX2), members of the SUPPRESSOR OF MAX2 1-LIKE (SMXL) protein family, and TOPLESS repressors (Arite et al. 2009; de Saint Germain et al. 2016; GomezRoldan et al. 2008; Hamiaux et al. 2012; Jiang et al. 2013; Marzec et al. 2016; Nakamura et al. 2013; Stirnberg et al. 2007; Umehara et al. 2008; Zhao et al. 2015; Zheng et al. 2016; Zhou et al. 2013). Upon strigolactone binding, the $\mathrm{SCF}^{\mathrm{MAX} 2}$ complex is anticipated to ubiquitinate the SMXL proteins to send them for proteasomal degradation, thereby activating downstream signaling (Jiang et al. 2013; Liang et al. 2016; Soundappan et al. 2015; Wang et al. 2015; Zhou et al. 2013). MAX2 is also essential for karrikin-induced seed germination (Nelson et al. 2011). However, rather than through D14, these smoke-derived germination stimulants (Waters 2017) are perceived by the D14 paralog D14-LIKE (D14L)/KARRIKIN INSENSITIVE 2 (KAI2), whereafter the $\mathrm{SCF}^{\mathrm{MAX} 2}$ complex is expected to ubiquitinate members of the SMXL family that differ from those targeted by the D14/SCF ${ }^{\mathrm{MAX} 2}$ complex (Guo et al. 2013; Soundappan et al. 2015; Stanga et al. 2013; Waters et al. 2012). The synthetic strigolactone analog, rac-GR24, which is ubiquitously used in strigolatone research, is a mixture of four isomers, of which two mimic natural strigolactones that activate mainly D14 signaling cascades and the other two preferentially activate KAI2 signaling (Scaffidi et al. 2014). Additionally, besides karrikins, also endogenous, still-unknown ligands for KAI2 are predictable, which are also mimicked by the rac-GR24 mixture (Conn and Nelson 2016).

Protein sequences of both MAX2 and D14 have been shown to match those of the translated genome of arbuscular mycorrhizal fungi, albeit with a low identity, implying that the ability to respond to strigolactones might have evolved separately in plants and fungi (Koltai 2014). Indeed, thus far, the identification of a real MAX2 or D14 ortholog has not been reported. Interestingly, inside rice (Oryza sativa) roots, the D14 paralog D14L is required to initiate the symbiosis with arbuscular mycorrhizal fungi (Gutjahr et al. 2015). Thus, karrikins or karrikin-like molecules might play a role in this symbiosis (Gutjahr et al. 2015). Whether these karrikin-like molecules are derived from the plant or from the fungus is currently unknown and would be interesting to investigate.

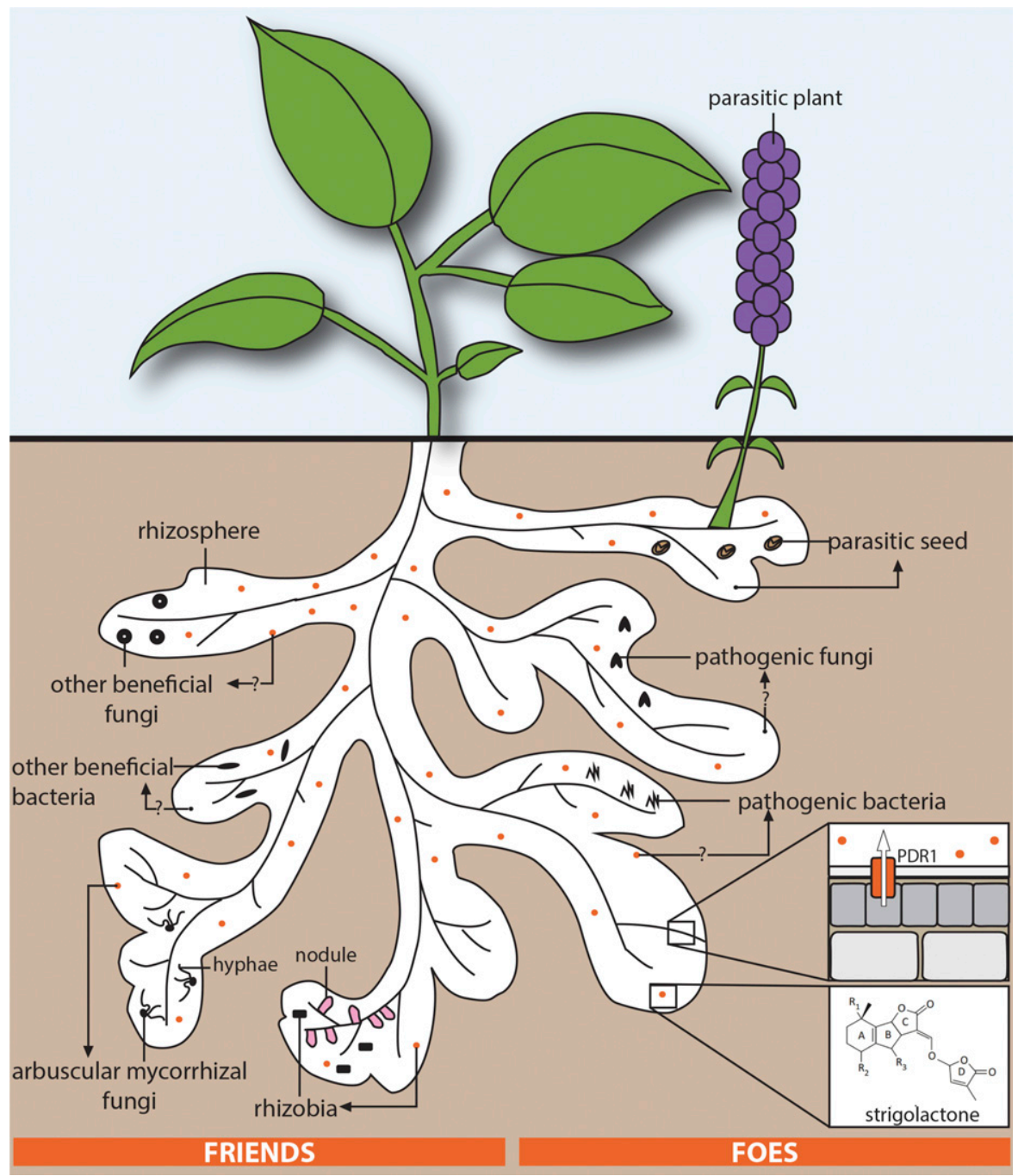

Fig. 1. Strigolactones as Janus-faced molecules in the rhizosphere. To interact with other organisms in the soil, plants secrete strigolactones in the rhizosphere through the strigolactone exporter PDR1. Rhizobia and arbuscular mycorrhiza have been shown to respond to strigolactones, favoring their interaction with the plant. However, parasitic weeds misuse the presence of strigolactones to recognize their hosts. 
Intriguingly, nonhost plants for arbuscular mycorrhizal fungi, such as Arabidopsis and Lupinus albus (white lupin), also produce and secrete strigolactones, hinting at a more general function as communication signals in other rhizosphere interactions. Whether or not strigolactones can influence the growth and branching of pathogenic fungi and oomycetes is not clear. Indeed, although no influence of rac-GR24 $\left[10^{-6} \mathrm{M}\right]$ or natural strigolactones on the branching pattern or germination of various root-colonizing beneficial and pathogenic fungal or oomycete species, including Fusarium oxysporum, has been observed (Blake et al. 2016; Decker et al. 2017; Foo et al. 2016; Steinkellner et al. 2007), treatment with rac-GR24 $\left[10^{-5} \mathrm{M}\right]$ strongly inhibits the growth of several pathogenic fungi, among which, again, Fusarium oxysporum (Decker et al. 2017; Dor et al. 2011). As most effects occur with the application of high concentrations of strigolactones $(50 \mu \mathrm{M})$, highly exceeding the levels found in the rhizosphere, strigolactones might probably only have little or no effect on the tested pathogens in nature.

In contrast, strigolactones might act as plant endogenous hormones to influence defense responses against fungal intruders. The strigolactone-deficient tomato Slccd8 RNA interference line has been shown to be more susceptible to the foliar fungal pathogens Botrytis cinerea and Alternaria alternata. This sensitivity correlates with reduced levels of the phytohormones jasmonate, salicylic acid, and abscisic acid, implying a role for strigolactones in plant defense (Torres-Vera et al. 2014). In agreement, strigolactone biosynthesis mutants of Physcomitrella patens have an enhanced disease susceptibility against various phytopathogenic fungi, possibly due to changes in defense responses rather than to direct effects on the pathogens (Decker et al. 2017). On the contrary, in pea (Pisum sativum), no changes in disease symptoms of Pythium irregulare or Fusarium oxysporum have been reported in various strigolactone synthesis and perception mutants (Blake et al. 2016; Foo et al. 2016) nor in the interaction between Arabidopsis strigolactone-deficient mutants and B. cinerea (Piisilä et al. 2015). Hence, the effects of strigolactones on diseases vary. Therefore, further research is required to better understand how strigolactones play a role in the interaction of plants with root-pathogenic fungi and oomycetes. Nevertheless, the impact of rac-GR24 on the colony growth of $B$. cinerea has been used to elucidate their mode of action. The analysis of $B$. cinerea mutants that are less sensitive to rac-GR24 suggests that responses rely on reactive oxygen species (ROS) and mitochondria (Belmondo et al. 2017).

\section{A cue for rhizobia and other bacteria.}

Besides the interaction between plants and arbuscular mycorrhizal fungi, another beneficial dialog in the rhizosphere is the rhizobia-legume symbiosis. Whereas the role of strigolactones in the communication with arbuscular mycorrhiza or parasitic plants is well-defined, their involvement in the communication with rhizobia is less clear and some discrepancies still exist. The symbiosis between legumes and rhizobia is generally referred to as nodulation, a process by which the soil bacteria invade the legume roots and form nodules in which the bacteria fix atmospheric nitrogen that is transferred to the plant in exchange for carbohydrates and a protective niche (Markmann and Parniske 2009).

Application of rac-GR24 positively affects the nodule number in Medicago sativa (alfalfa), with an increase of 30 to $40 \%$ compared with untreated controls (Soto et al. 2010). In agreement with these results, transgenic Lotus japonicus plants silenced for the strigolactone biosynthesis gene CAROTENOID CLEAVAGE DIOXYGENASE 7 (LjCCD7) develop approximately $20 \%$ fewer nodules than the control plants, without alterations in nodule development or morphology (Liu et al. 2013).
Also in ramosus 1 ( $r m s \mathrm{l}$ ) mutants of pea, defective in the strigolactone biosynthesis gene PsCCD8, approximately $40 \%$ fewer nodules are produced, whereas treatment with $\mathrm{rac}$-GR24 restores this hyponodulation phenotype (Foo and Davies 2011). In addition, the nodule number decreases significantly in rms $5 \mathrm{mu}-$ tants, which are defective in PSCCD7 (Foo et al. 2013), but it increases in the strigolactone-insensitive rms 4 mutant that is affected in the Arabidopsis ortholog MAX2 involved in strigolactone signaling (Foo and Davies 2011). Although this observation contrasts with the data obtained for the rms 1 mutants and the $\mathrm{LjCCD} 7$ lines, $r a c$-GR24 treatment could affect the nodule number in a concentration-dependent manner in Medicago truncatula (barrel medic), with a stimulating effect at low racGR24 concentrations and a negative effect at high concentrations (De Cuyper et al. 2015).

As treatment with rac-GR24 has no impact on bacterial growth, nodulation factor production, or bacterial calcium spiking, rac-GR24 has been suggested to act on the plant side as a plant hormone and not directly on the bacteria, which is in contrast to their role as rhizosphere signaling molecules in the interaction with arbuscular mycorrhizal fungi (Moscatiello et al. 2010; Soto et al. 2010). However, the crystal structure of the rice strigolactone receptor D14 resembles that of the Bacillus subtilis RsbQ protein that is a stress-response regulator with hydrolase activity (Kagiyama et al. 2013), indicating that strigolactones might influence rhizobial cellular activity in a manner different from that tested above. Indeed, a role for strigolactones has been implied in the chemical dialog between plants and rhizobia (Peláez-Vico et al. 2016; Tambalo et al. 2014). For instance, extracts of the moss Physcomitrella patens stimulate the swarming motility, i.e., a rapid coordinated movement of bacteria across a surface, in Rhizobium leguminosarum, whereas this promoting effect is reduced by extracts from the strigolactone-deficient strain Physcomitrella patens Ppccd8 (Peláez-Vico et al. 2016; Tambalo et al. 2014). In addition, rac-GR24 triggers the swarming motility of Sinorhizobium meliloti in a dose-dependent manner (PeláezVico et al. 2016). Both studies also describe a correlation between increased surface motility and a stimulation of flagellin flaA gene expression in response to either moss extracts or racGR24, further providing evidence that rhizobia can, indeed, perceive and respond to strigolactones. Whether or not this effect causes the observed changes in nodule number needs further investigation, because changes in rhizobial swarming have been shown to influence nodulation in some cases and not in others (Bernabéu-Roda et al. 2015; Caetano-Anollés et al. 1988; Soto et al. 2002; Wei and Bauer 1999). Taken together, strigolactones might, besides controlling the optimal nodule number as a plant hormone, also act as host detection cues for rhizobia in the rhizosphere, by stimulating bacterial swarming motility and, very probably, the rhizobia-legume symbiosis as well. However, the ecological relevance of this swarming behavior is poorly described and needs to be investigated to better understand the impact on nodulation.

With the exception of their role in communication with rhizobia, no function in dialog with other soil bacteria has been reported for strigolactones until now, but strigolactones have been linked to interaction with different bacteria in the shoot. For instance, in Arabidopsis, the MAX2 protein, implicated in strigolactone signaling, has contributed to plant disease resistance against the bacterial pathogens Pectobacterium carotovorum and Pseudomonas syringae. The reason for the increased susceptibility to both pathogens of the $\max 2$ mutants might be an enhanced stomatal conductance, promoting pathogen entry into the plant apoplast, a decreased tolerance to apoplastic ROS, and alterations in hormonal balances (Piisilä et al. 2015). Moreover, because both strigolactone biosynthesis and signaling mutants display 
enhanced symptoms upon infection with the pathogenic Rhodococcus fascians, strigolactones have also been proposed to act as a defense mechanism against leafy gall syndrome indirectly, by inhibiting the outgrowth of newly formed shoots from the leafy galls (Stes et al. 2015). Future studies need to clarify whether strigolactones can also directly interact with pathogenic bacteria and not only indirectly play a role in defense. Interestingly, in rice, grassy stunt disease, characterized by excessive tillering, provoked by the grassy stunt virus is correlated with the suppression of strigolactone biosynthesis and signaling genes (Satoh et al. 2013).

In silico analyses of the promoter regions of strigolactone biosynthesis genes have also revealed a role in biotic stress responses (Marzec and Muszynska 2015). Indeed, in Arabidopsis and rice, cis-regulatory motifs involved in defense reactions against viruses, bacteria, and fungi have been identified in the promoters of strigolactone biosynthesis genes (Dong et al. 2003; Gutha and Reddy 2008; Kalde et al. 2003; Liu et al. 2007). As a result, the strigolactone biosynthesis pathway could be regulated by specific biotic stress-related transcription factors to protect the plant. To gain more insight into the importance of strigolactones during biotic stress responses, comparative metagenomics analyses that combine different genotypes (such as strigolactone-deficient and -overproducing lines) and various pathogens need to be done.

\section{A cue for parasitic plants.}

To prevent germination in the absence of suitable host roots, parasitic plants have established the need for germination stimulants released by their specific hosts. Strigolactones stimulate the germination of Striga spp. (witchweeds), Orobanche spp., Phelipanche spp., and Alectra spp. (broomrapes), all rootparasitic weeds that belong to the Orobanchaceae family and are considered as the most damaging plants for agriculture worldwide (Parker 2009). Indeed, Striga spp. parasitize important food crops such as sorghum (Sorghum bicolor), maize, millet (Pennisetum glaucum), and rice, with enormous yield losses in sub-Saharan Africa and parts of Asia as a consequence (Parker 2009). Orobanche and Phelipanche species are a major pest for crops growing under a more temperate climate, e.g., tomato, tobacco, carrot (Daucus carota), rapeseed (Brassica napus), and sunflower (Helianthus sp.), and cause huge problems in Europe, the Middle East, and North Africa. The millions of hectares infested by these parasitic plants generate enormous losses in crop yield, estimated to correspond with billions of US\$ (Parker 2009).

The survival and development of most parasitic plants depend fully on the nearby presence of a host plant, because of the slight, or lack of, photosynthetic capacity and of the small storage reserves of their thousands of small seeds $(0.2 \mathrm{~mm}$ in diameter). The seeds can remain dormant (up to 10 years and more) until they sense specific host-derived germination stimulants, such as strigolactones, that ensure that the parasite germinates only when a host plant is within reach (Cardoso et al. 2011; Tsuchiya and McCourt 2009).

Parasitic plants germinate already in the presence of nanomolar concentrations of rac-GR24. Nevertheless, the molecular mechanisms of strigolactone perception and induction of seed germination in parasitic plants are now emerging. In Arabidopsis, D14 is characterized as the strigolactone receptor, whereas its paralog, KAI2, is involved in the perception of smoke-derived karrikin molecules (Waters et al. 2012). However, strigolactone perception by the parasites is most probably mediated through a divergent KAI 2 receptor, from which the binding pocket has evolved to bind to strigolactones instead of karrikins (Conn et al. 2015). Interestingly, because the divergent parasitic plant KAI2 could complement the
Arabidopsis kai2 mutant and make it highly sensitive to racGR24-mediated germination but not to karrikin-induced germination, the other members of the signaling complex, in addition to KAI2, are expected to be conserved between parasitic and nonparasitic plants (Conn and Nelson 2016; Toh et al. 2015; Xu et al. 2016).

To be responsive to strigolactones, parasitic seeds need to pass through a conditioning period for a minimum of 4 days under appropriate humidity, temperature, and oxygen conditions (Joel et al. 2012; Lechat et al. 2012). This period is characterized by water uptake via the micropyle, followed by imbibition of the seed and two essential consecutive reductions in abscisic acid levels. The first one is caused by exudation of abscisic acid, whereas the second one results from strigolactone perception. Indeed, the abscisic acid catabolic gene CYP707A1 catalyzes the breakdown of abscisic acid, and treatment with rac-GR24 results in a strong and fast upregulation of this gene in Phelipanche ramosa, whereas treatment with a combination of rac-GR24 and CYP707A1 inhibitors blocks $C Y P 707 A 1$ gene expression and prevents germination (Lechat et al. 2012, 2015). Interestingly, this $C Y P 707 A 1$ induction depends on the DNA methylation level of its promoter as well as on the $26 \mathrm{~S}$ proteasome, indicating that $\mathrm{rac}$-GR24 can have an influence at both the RNA and protein levels (Lechat et al. 2012). After germination, the root emerges from the seed coat and forms a specialized structure, the haustorium, which fastens and penetrates the host root. Subsequently, this organ is linked with the host vascular system, from which water, carbon, and nutrients are obtained and are used to develop shoots and flowers that can produce seeds again (Cardoso et al. 2011; Press and Phoenix 2005). As a result of this interaction, the host plant will become severely weakened, growth will be impaired, and eventually the plant will die.

Different approaches to eliminate parasitic weeds from agricultural lands have been explored, such as soil treatment with synthetic strigolactones, use of mutants with limited strigolactone production or exudation, and cultivation of trap-andcatch crops, i.e., nonhost species that produce germination stimulants that lead to a suicidal germination of the parasitic plant seeds in the soil. By increasing the strigolactone production and exudation or by switching to more active strigolactone types, more effective trap crops might be created (Waters et al. 2017). Indeed, the search for cost-effective, practical, and targeted strategies still continues (Vurro et al. 2016). Interestingly, a well-established symbiosis with arbuscular mycorrhiza reduces strigolactone levels in the plant as a negative feedback mechanism to avoid excessive colonization (García-Garrido et al. 2009; López-Ráez et al. 2011; Mabrouk et al. 2007). Although it is still unknown whether this reduction in strigolactone levels is due to a direct effect on the strigolactone biosynthesis itself or indirectly through the improvement of the plant nutritional status by the symbiosis, this decrease in strigolactone levels might aid the plant to become less sensitive against parasitic plant infections. Moreover, other soil microorganisms can assist in preventing the germination of parasitic plants by acting as a physiological root barrier and by reducing the strigolactone content in the rhizosphere. For instance, the beneficial fungus Trichoderma harzianum detects and metabolizes strigolactones and might be of great value as a bioherbicide in parasitic weed management (Boari et al. 2016). In addition, different plant species produce a different blend of strigolactones, possibly with an impact on the sensitivities to parasitic plants (Ćavar et al. 2015). Modification of the strigolactone secretion profile toward a blend to which parasitic plants are insensitive but arbuscular mycorrhizal fungi are not is a possible route to reduce parasitic plant infestations (Yoneyama et al. 2015). 


\section{Conclusion.}

To establish an interaction between plants and surrounding organisms, a complex chemical dialog takes place, in which both partners exchange information by exudation and perception of different molecules. Here, we discussed strigolactones as plant-derived communication signals. Thus far, strigolactones are well-established communication molecules during arbuscular mycorrhization. As parasitic weeds have evolved to exploit the presence of strigolactones to recognize and infect their host, strigolactones behave as Janus-faced (god with two faces) molecules in the rhizosphere, i.e., they present the plant with a challenge, namely bonding with their friends while hiding from their foes (Fig. 1). Whether they also serve as plant signals toward other beneficial or detrimental rhizospheric microbes is currently less clear. Until now, single models have been studied, consisting of one plant in relation to one microbe. These studies should be strengthened but should go hand-in-hand with microbial community analysis by the application of various "omics" approaches that allow in-depth insights into the microbial diversity of the rhizosphere (Dessaux et al. 2016). With the different strigolactone mutants at hand, precise surveys can be made of the effects of strigolactone exudation on the microbial life in the rhizosphere. From these studies, key interactions influenced by strigolactones will be identified that should be further investigated with dedicated studies to tell us how broadly strigolactones impact on the microbial life in the rhizosphere.

\section{ACKNOWLEDGMENTS}

This work was supported by the European Cooperation on Science and Technology (COST action FA1206). C. De Cuyper was a predoctoral fellow of the Research Foundation-Flanders.

\section{LITERATURE CITED}

Abe, S., Sado, A., Tanaka, K., Kisugi, T., Asami, K., Ota, S., Kim, H. I., Yoneyama, K., Xie, X., Ohnishi, T., Seto, Y., Yamaguchi, S., Akiyama, K., Yoneyama, K., and Nomura, T. 2014. Carlactone is converted to carlactonoic acid by MAX1 in Arabidopsis and its methyl ester can directly interact with AtD14 in vitro. Proc. Natl. Acad. Sci. U.S.A. 111: 18084-18089.

Akiyama, K., Matsuzaki, K.-i., and Hayashi, H. 2005. Plant sesquiterpenes induce hyphal branching in arbuscular mycorrhizal fungi. Nature 435: 824-827.

Akiyama, K., Ogasawara, S., Ito, S., and Hayashi, H. 2010. Structural requirements of strigolactones for hyphal branching in AM fungi. Plant Cell Physiol. 51:1104-1117.

Al-Babili, S., and Bouwmeester, H. J. 2015. Strigolactones, a novel carotenoid-derived plant hormone. Annu. Rev. Plant Biol. 66:161-186.

Alder, A., Jamil, M., Marzorati, M., Bruno, M., Vermathen, M., Bigler, P., Ghisla, S., Bouwmeester, H., Beyer, P., and Al-Babili, S. 2012. The path from $\beta$-carotene to carlactone, a strigolactone-like plant hormone. Science 335:1348-1351.

Arite, T., Umehara, M., Ishikawa, S., Hanada, A., Maekawa, M., Yamaguchi, S., and Kyozuka, J. 2009. d14, a strigolactone-insensitive mutant of rice, shows an accelerated outgrowth of tillers. Plant Cell Physiol. 50:1416-1424.

Bais, H. P., Weir, T. L., Perry, L. G., Gilroy, S., and Vivanco, J. M. 2006. The role of root exudates in rhizosphere interactions with plants and other organisms. Annu. Rev. Plant Biol. 57:233-266.

Belmondo, S., Marschall, R., Tudzynski, P., López Ráez, J. A., Artuso, E., Prandi, C., and Lanfranco, L. 2017. Identification of genes involved in fungal responses to strigolactones using mutants from fungal pathogens. Curr. Genet. 63:201-213.

Bernabéu-Roda, L., Calatrava-Morales, N., Cuéllar, V., and Soto, M. J. 2015. Characterization of surface motility in Sinorhizobium meliloti: Regulation and role in symbiosis. Symbiosis 67:79-90.

Besserer, A., Bécard, G., Jauneau, A., Roux, C., and Séjalon-Delmas, N. 2008. GR24, a synthetic analog of strigolactones, stimulates the mitosis and growth of the arbuscular mycorrhizal fungus Gigaspora rosea by boosting its energy metabolism. Plant Physiol. 148:402-413.

Besserer, A., Puech-Pagès, V., Kiefer, P., Gomez-Roldan, V., Jauneau, A., Roy, S., Portais, J.-C., Roux, C., Bécard, G., and Séjalon-Delmas, N.
2006. Strigolactones stimulate arbuscular mycorrhizal fungi by activating mitochondria. PLoS Biol. 4:e226.

Blake, S. N., Barry, K. M., Gill, W. M., Reid, J. B., and Foo, E. 2016. The role of strigolactones and ethylene in disease caused by Pythium irregulare. Mol. Plant Pathol. 17:680-690.

Boari, A., Ciasca, B., Pineda-Martos, R., Lattanzio, V. M. T., Yoneyama, K., and Vurro, M. 2016. Parasitic weed management by using strigolactonedegrading fungi. Pest Manag. Sci. 72:2043-2047.

Booker, J., Auldridge, M., Wills, S., McCarty, D., Klee, H., and Leyser, O. 2004. MAX3/CCD7 is a carotenoid cleavage dioxygenase required for the synthesis of a novel plant signaling molecule. Curr. Biol. 14: 1232-1238.

Booker, J., Sieberer, T., Wright, W., Williamson, L., Willett, B., Stirnberg, P., Turnbull, C., Srinivasan, M., Goddard, P., and Leyser, O. 2005. MAXI encodes a cytochrome $\mathrm{P} 450$ family member that acts downstream of $M A X 3 / 4$ to produce a carotenoid-derived branch-inhibiting hormone. Dev. Cell 8:443-449.

Borghi, L., Kang, J., Ko, D., Lee, Y., and Martinoia, E. 2015. The role of ABCG-type ABC transporters in phytohormone transport. Biochem. Soc. Trans. 43:924-930.

Borghi, L., Liu, G.-W., Emonet, A., Kretzschmar, T., and Martinoia, E. 2016. The importance of strigolactone transport regulation for symbiotic signaling and shoot branching. Planta 243:1351-1360.

Brewer, P. B., Yoneyama, K., Filardo, F., Meyers, E., Scaffidi, A., Frickey, T., Akiyama, K., Seto, Y., Dun, E. A., Cremer, J. E., Kerr, S. C., Waters, M. T., Flematti, G. R., Mason, M. G., Weiller, G., Yamaguchi, S., Nomura, T., Smith, S. M., Yoneyama, K., and Beveridge, C. A. 2016. LATERAL BRANCHING OXIDOREDUCTASE acts in the final stages of strigolactone biosynthesis in Arabidopsis. Proc. Natl. Acad. Sci. U.S.A. 113:6301-6306.

Bruno, M., Vermathen, M., Alder, A., Wüst, F., Schaub, P., van der Steen, R., Beyer, P., Ghisla, S., and Al-Babili, S. 2017. Insights into the formation of carlactone from in-depth analysis of the CCD8-catalyzed reactions. FEBS Lett. 591:792-800.

Buée, M., De Boer, W., Martin, F., van Overbeek, L., and Jurkevitch, E. 2009. The rhizosphere zoo: An overview of plant-associated communities of microorganisms, including phages, bacteria, archaea, and fungi, and of some of their structuring factors. Plant Soil 321:189-212.

Buee, M., Rossignol, M., Jauneau, A., Ranjeva, R., and Bécard, G. 2000. The pre-symbiotic growth of arbuscular mycorrhizal fungi is induced by a branching factor partially purified from plant root exudates. Mol Plant-Microbe Interact 13:693-698.

Caetano-Anollés, G., Wall, L. G., De Micheli, A. T., Macchi, E. M., Bauer, W. D., and Favelukes, G. 1988. Role of motility and chemotaxis in efficiency of nodulation by Rhizobium meliloti. Plant Physiol. 86: 1228-1235

Cardoso, C., Ruyter-Spira, C., and Bouwmeester, H. J. 2011. Strigolactones and root infestation by plant-parasitic Striga, Orobanche and Phelipanche spp. Plant Sci. 180:414-420.

Ćavar, S., Zwanenburg, B., and Tarkowski, P. 2015. Strigolactones: Occurrence, structure, and biological activity in the rhizosphere. Phytochem. Rev. 14:691-711.

Conn, C. E., Bythell-Douglas, R., Neumann, D., Yoshida, S., Whittington, B., Westwood, J. H., Shirasu, K., Bond, C. S., Dyer, K. A., and Nelson, D. C. 2015. Convergent evolution of strigolactone perception enabled host detection in parasitic plants. Science 349:540-543.

Conn, C. E., and Nelson, D. C. 2016. Evidence that KARRIKININSENSITIVE2 (KAI2) receptors may perceive an unknown signal that is not karrikin or strigolactone. Front. Plant Sci. 6:1219.

Cook, C. E., Whichard, L. P., Turner, B., Wall, M. E., and Egley, G. H. 1966. Germination of witchweed (Striga lutea Lour.): Isolation and properties of a potent stimulant. Science 154:1189-1190.

De Cuyper, C., Fromentin, J., Yocgo, R. E., De Keyser, A., Guillotin, B., Kunert, K., Boyer, F.-D., and Goormachtig, S. 2015. From lateral root density to nodule number, the strigolactone analogue GR24 shapes the root architecture of Medicago truncatula. J. Exp. Bot. 66:137-146. [Corrigendum J. Exp. Bot. 66:4091].

de Saint Germain, A., Clavé, G., Badet-Denisot, M.-A., Pillot, J.-P., Cornu, D., Le Caer, J.-P., Burger, M., Pelissier, F., Retailleau, P., Turnbull, C., Bonhomme, S., Chory, J., Rameau, C., and Boyer, F.-D. 2016. An histidine covalent receptor and butenolide complex mediates strigolactone perception. Nat. Chem. Biol. 12:787-794.

Decker, E. L., Alder, A., Hunn, S., Ferguson, J., Lehtonen, M. T., Scheler, B., Kerres, K. L., Wiedemann, G., Safavi-Rizi, V., Nordzieke, S. Balakrishna, A., Baz, L., Avalos, J., Valkonen, J. P. T., Reski, R., and AlBabili, S. 2017. Strigolactone biosynthesis is evolutionarily conserved, regulated by phosphate starvation and contributes to resistance against 
phytopathogenic fungi in a moss, Physcomitrella patens. New Phytol. Published online. 10.1111/nph.14506

Delaux, P.-M., Xie, X., Timme, R. E., Puech-Pages, V., Dunand, C., Lecompte, E., Delwiche, C. F., Yoneyama, K., Bécard, G., and SéjalonDelmas, N. 2012. Origin of strigolactones in the green lineage. New Phytol. 195:857-871.

Dessaux, Y., Grandclément, C., and Faure, D. 2016. Engineering the rhizosphere. Trends Plant Sci. 21:266-278.

Dong, J., Chen, C., and Chen, Z. 2003. Expression profiles of the Arabidopsis WRKY gene superfamily during plant defense response. Plant Mol. Biol. 51:21-37.

Dor, E., Joel, D. M., Kapulnik, Y., Koltai, H., and Hershenhorn, J. 2011. The synthetic strigolactone GR24 influences the growth pattern of phytopathogenic fungi. Planta 234:419-427.

Foo, E., Blake, S. N., Fisher, B. J., Smith, J. A., and Reid, J. B. 2016. The role of strigolactones during plant interactions with the pathogenic fungus Fusarium oxysporum. Planta 243:1387-1396.

Foo, E., and Davies, N. W. 2011. Strigolactones promote nodulation in pea. Planta 234:1073-1081.

Foo, E., Yoneyama, K., Hugill, C. J., Quittenden, L. J., and Reid, J. B. 2013. Strigolactones and the regulation of pea symbioses in response to nitrate and phosphate deficiency. Mol. Plant 6:76-87.

García-Garrido, J. M., Lendzemo, V., Castellanos-Morales, V., Steinkellner, S., and Vierheilig, H. 2009. Strigolactones, signals for parasitic plants and arbuscular mycorrhizal fungi. Mycorrhiza 19:449-459.

Giovannetti, M., Sbrana, C., Avio, L., Citernesi, A. S., and Logi, C. 1993 Differential hyphal morphogenesis in arbuscular mycorrhizal fungi during pre-infection stages. New Phytol. 125:587-593.

Giovannetti, M., Sbrana, C., Citernesi, A. S., and Avio, L. 1996. Analysis of factors involved in fungal recognition responses to host-derived signals by arbuscular mycorrhizal fungi. New Phytol. 133:65-71.

Giovannetti, M., Sbrana, C., and Logi, C. 1994. Early processes involved in host recognition by arbuscular mycorrhizal fungi. New Phytol. 127: 703-709.

Gomez-Roldan, V., Fermas, S., Brewer, P. B., Puech-Pagès, V., Dun, E. A., Pillot, J.-P., Letisse, F., Matusova, R., Danoun, S., Portais, J.-C., Bouwmeester, H., Bécard, G., Beveridge, C. A., Rameau, C., and Rochange, S. F. 2008. Strigolactone inhibition of shoot branching. Nature 455:189-194.

Gomez-Roldan, V., Roux, C., Girard, D., Bécard, G., and Puech-Pagés, V. 2007. Strigolactones: Promising plant signals. Plant Signal. Behav. 2:163-164.

Guo, Y., Zheng, Z., La Clair, J. J., Chory, J., and Noel, J. P. 2013. Smokederived karrikin perception by the $\alpha / \beta$-hydrolase KAI 2 from Arabidopsis. Proc. Natl. Acad. Sci. U.S.A. 110:8284-8289.

Gutha, L. R., and Reddy, A. R. 2008. Rice DREB1B promoter shows distinct stress-specific responses, and the overexpression of cDNA in tobacco confers improved abiotic and biotic stress tolerance. Plant Mol. Biol. 68:533-555

Gutjahr, C., Gobbato, E., Choi, J., Riemann, M., Johnston, M. G., Summers, W., Carbonnel, S., Mansfield, C., Yang, S.-Y., Nadal, M. Acosta, I., Takano, M., Jiao, W.-B., Schneeberger, K., Kelly, K. A., and Paszkowski, U. 2015. Rice perception of symbiotic arbuscular mycorrhizal fungi requires the karrikin receptor complex. Science 350:1521-1524.

Hamiaux, C., Drummond, R. S. M., Janssen, B. J., Ledger, S. E., Cooney, J. M., Newcomb, R. D., and Snowden, K. C. 2012. DAD2 is an $\alpha / \beta$ hydrolase likely to be involved in the perception of the plant branching hormone, strigolactone. Curr. Biol. 22:2032-2036.

Jiang, L., Liu, X., Xiong, G., Liu, H., Chen, F., Wang, L., Meng, X., Liu, G., Yu, H., Yuan, Y., Yi, W., Zhao, L., Ma, H., He, Y., Wu, Z., Melcher, K., Qian, Q., Xu, H. E., Wang, Y., and Li, J. 2013. DWARF 53 acts as a repressor of strigolactone signalling in rice. Nature 504:401-405.

Joel, D. M., Bar, H., Mayer, A. M., Plakhine, D., Ziadne, H., Westwood, J. H., and Welbaum, G. E. 2012. Seed ultrastructure and water absorption pathway of the root-parasitic plant Phelipanche aegyptiaca (Orobanchaceae). Ann. Bot. (Lond.) 109:181-195.

Kagiyama, M., Hirano, Y., Mori, T., Kim, S.-Y., Kyozuka, J., Seto, Y., Yamaguchi, S., and Hakoshima, T. 2013. Structures of D14 and D14L in the strigolactone and karrikin signaling pathways. Genes Cells 18: 147-160.

Kalde, M., Barth, M., Somssich, I. E., and Lippok, B. 2003. Members of the Arabidopsis WRKY group III transcription factors are part of different plant defense signaling pathways. Mol. Plant-Microbe Interact 16: 295-305.

Kang, J., Hwang, J.-U., Lee, M., Kim, Y.-Y., Assmann, S. M., Martinoia, E., and Lee, Y. 2010. PDR-type ABC transporter mediates cellular uptake of the phytohormone abscisic acid. Proc. Natl. Acad. Sci. U.S.A. 107: 2355-2360.
Kohlen, W., Charnikhova, T., Lammers, M., Pollina, T., Tóth, P., Haider, I., Pozo, M. J., de Maagd, R. A., Ruyter-Spira, C., Bouwmeester, H. J., and López-Ráez, J. A. 2012. The tomato CAROTENOID CLEAVAGE DIOXYGENASE8 (SICCD8) regulates rhizosphere signaling, plant architecture and affects reproductive development through strigolactone biosynthesis. New Phytol. 196:535-547.

Kohlen, W., Charnikhova, T., Liu, Q., Bours, R., Domagalska, M. A., Beguerie, S., Verstappen, F., Leyser, O., Bouwmeester, H., and RuyterSpira, C. 2011. Strigolactones are transported through the xylem and play a key role in shoot architectural response to phosphate deficiency in nonarbuscular mycorrhizal host Arabidopsis. Plant Physiol. 155: 974-987.

Koltai, H. 2014. Implications of non-specific strigolactone signaling in the rhizosphere. Plant Sci. 225:9-14

Koltai, H., LekKala, S. P., Bhattacharya, C., Mayzlish-Gati, E., Resnick, N., Wininger, S., Dor, E., Yoneyama, K., Yoneyama, K., Hershenhorn, J., Joel, D. M., and Kapulnik, Y. 2010. A tomato strigolactone-impaired mutant displays aberrant shoot morphology and plant interactions. J. Exp. Bot. 61:1739-1749.

Kretzschmar, T., Kohlen, W., Sasse, J., Borghi, L., Schlegel, M., Bachelier, J. B., Reinhardt, D., Bours, R., Bouwmeester, H. J., and Martinoia, E. 2012. A petunia ABC protein controls strigolactone-dependent symbiotic signalling and branching. Nature 483:341-344.

Lechat, M.-M., Brun, G., Montiel, G., Véronési, C., Simier, P., Thoiron, S. Pouvreau, J.-B., and Delavault, P. 2015. Seed response to strigolactone is controlled by abscisic acid-independent DNA methylation in the obligate root parasitic plant, Phelipanche ramosa L. Pomel. J. Exp. Bot. 66: 3129-3140.

Lechat, M.-M., Pouvreau, J.-B., Péron, T., Gauthier, M., Montiel, G., Véronési, C., Todoroki, Y., Le Bizec, B., Monteau, F., Macherel, D., Simier, P., Thoiron, S., and Delavault, P. 2012. PrCYP707A1, an ABA catabolic gene, is a key component of Phelipanche ramosa seed germination in response to the strigolactone analogue GR24. J. Exp. Bot. 63:5311-5322.

Leyser, O. 2008. Strigolactones and shoot branching: A new trick for a young dog. Dev. Cell 15:337-338.

Liang, Y., Ward, S., Li, P., Bennett, T., and Leyser, O. 2016. SMAX1LIKE7 signals from the nucleus to regulate shoot development in Arabidopsis via partially EAR motif-independent mechanisms. Plant Cell 28:1581-1601.

Lin, H., Wang, R., Qian, Q., Yan, M., Meng, X., Fu, Z., Yan, C., Jiang, B., $\mathrm{Su}, \mathrm{Z}$., Li, J., and Wang, Y. 2009. DWARF27, an iron-containing protein required for the biosynthesis of strigolactones, regulates rice tiller bud outgrowth. Plant Cell 21:1512-1525.

Liu, J., Novero, M., Charnikhova, T., Ferrandino, A., Schubert, A., RuyterSpira, C., Bonfante, P., Lovisolo, C., Bouwmeester, H. J., and Cardinale, F. 2013. CAROTENOID CLEAVAGE DIOXYGENASE 7 modulates plant growth, reproduction, senescence, and determinate nodulation in the model legume Lotus japonicus. J. Exp. Bot. 64:1967-1981.

Liu, X., Bai, X., Wang, X., and Chu, C. 2007. OsWRKY71, a rice transcription factor, is involved in rice defense response. J. Plant Physiol. 164:969-979.

López-Ráez, J. A., Charnikhova, T., Fernández, I., Bouwmeester, H., and Pozo, M. J. 2011. Arbuscular mycorrhizal symbiosis decreases strigolactone production in tomato. J. Plant Physiol. 168:294-297.

Mabrouk, Y., Simier, P., Delavault, P., Delgrange, S., Sifi, B., Zourgui, L., and Belhadj, O. 2007. Molecular and biochemical mechanisms of defence induced in pea by Rhizobium leguminosarum against Orobanche crenata. Weed Res. 47:452-460.

Markmann, K., and Parniske, M. 2009. Evolution of root endosymbiosis with bacteria: How novel are nodules? Trends Plant Sci. 14:77-86.

Marzec, M., Gruszka, D., Tylec, P., and Szarejko, I. 2016. Identification and functional analysis of the $\mathrm{HvDl4}$ gene involved in strigolactone signaling in Hordeum vulgare. Physiol. Plant. 158:341-355.

Marzec, M., and Muszynska, A. 2015. In silico analysis of the genes encoding proteins that are involved in the biosynthesis of the RMS/MAX/D pathway revealed new roles of strigolactones in plants. Int. J. Mol. Sci. 16: 6757-6782.

Mori, N., Nishiuma, K., Sugiyama, T., Hayashi, H., and Akiyama, K. 2016. Carlactone-type strigolactones and their synthetic analogues as inducers of hyphal branching in arbuscular mycorrhizal fungi. Phytochemistry 130:90-98.

Moscatiello, R., Squartini, A., Mariani, P., and Navazio, L. 2010. Flavonoid-induced calcium signalling in Rhizobium leguminosarum bv. viciae. New Phytol. 188:814-823.

Nakamura, H., Xue, Y.-L., Miyakawa, T., Hou, F., Qin, H.-M., Fukui, K., Shi, X., Ito, E., Ito, S., Park, S.-H., Miyauchi, Y., Asano, A., Totsuka, N., Ueda, T., Tanokura, M., and Asami, T. 2013. Molecular mechanism of strigolactone perception by DWARF14. Nat. Commun. 4:2613. 
Nelson, D. C., Scaffidi, A., Dun, E. A., Waters, M. T., Flematti, G. R., Dixon, K. W., Beveridge, C. A., Ghisalberti, E. L., and Smith, S. M. 2011. F-box protein MAX2 has dual roles in karrikin and strigolactone signaling in Arabidopsis thaliana. Proc. Natl. Acad. Sci. U.S.A. 108:8897-8902.

Parker, C. 2009. Observations on the current status of Orobanche and Striga problems worldwide. Pest Manag. Sci. 65:453-459.

Peláez-Vico, M. A., Bernabéu-Roda, L., Kohlen, W., Soto, M. J., and López-Ráez, J. A. 2016. Strigolactones in the Rhizobium-legume symbiosis: Stimulatory effect on bacterial surface motility and downregulation of their levels in nodulated plants. Plant Sci. 245:119-127.

Piisilä, M., Keceli, M. A., Brader, G., Jakobson, L., Jõesaar, I., Sipari, N., Kollist, H., Palva, E. T., and Kariola, T. 2015. The F-box protein MAX2 contributes to resistance to bacterial phytopathogens in Arabidopsis thaliana. BMC Plant Biol. 15:53.

Press, M. C., and Phoenix, G. K. 2005. Impacts of parasitic plants on natural communities. New Phytol. 166:737-751.

Raaijmakers, J. M., Paulitz, T. C., Steinberg, C., Alabouvette, C., and MoënneLoccoz, Y. 2009. The rhizosphere: A playground and battlefield for soilborne pathogens and beneficial microorganisms. Plant Soil 321:341-361.

Remy, W., Taylor, T. N., Hass, H., and Kerp, H. 1994. Four hundredmillion-year-old vesicular arbuscular mycorrhizae. Proc. Natl. Acad. Sci. U.S.A. 91:11841-11843.

Sasse, J., Simon, S., Gübeli, C., Liu, G.-W., Cheng, X., Friml, J., Bouwmeester, H., Martinoia, E., and Borghi, L. 2015. Asymmetric localizations of the $\mathrm{ABC}$ transporter PaPDR1 trace paths of directional strigolactone transport. Curr. Biol. 25:647-655.

Satoh, K., Yoneyama, K., Kondoh, H., Shimizu, T., Sasaya, T., Choi, I.-R., Yoneyama, K., Omura, T., and Kikuchi, S. 2013. Relationship between gene responses and symptoms induced by Rice grassy stunt virus. Front. Microbiol. 4:313.

Scaffidi, A., Waters, M. T., Sun, Y. K., Skelton, B. W., Dixon, K. W., Ghisalberti, E. L., Flematti, G. R., and Smith, S. M. 2014. Strigolactone hormones and their stereoisomers signal through two related receptor proteins to induce different physiological responses in Arabidopsis. Plant Physiol. 165:1221-1232.

Seto, Y., Sado, A., Asami, K., Hanada, A., Umehara, M., Akiyama, K., and Yamaguchi, S. 2014. Carlactone is an endogenous biosynthetic precursor for strigolactones. Proc. Natl. Acad. Sci. U.S.A. 111:1640-1645.

Sharda, J. N., and Koide, R. T. 2008. Can hypodermal passage cell distribution limit root penetration by mycorrhizal fungi? New Phytol. 180:696-701.

Soto, M. J., Fernández-Aparicio, M., Castellanos-Morales, V., GarcíaGarrido, J. M., Ocampo, J. A., Delgado, M. J., and Vierheilig, H. 2010. First indications for the involvement of strigolactones on nodule formation in alfalfa (Medicago sativa). Soil Biol. Biochem. 42:383-385.

Soto, M. J., Fernández-Pascual, M., Sanjuan, J., and Olivares, J. 2002. A fadD mutant of Sinorhizobium meliloti shows multicellular swarming migration and is impaired in nodulation efficiency on alfalfa roots. Mol. Microbiol. 43:371-382.

Soundappan, I., Bennett, T., Morffy, N., Liang, Y., Stanga, J. P., Abbas, A., Leyser, O., and Nelson, D. C. 2015. SMAX1-LIKE/D53 family members enable distinct MAX2-dependent responses to strigolactones and karrikins in Arabidopsis. Plant Cell 27:3143-3159.

Stanga, J. P., Smith, S. M., Briggs, W. R., and Nelson, D. C. 2013. SUPPRESSOR OF MORE AXILLARY GROWTH2 1 controls seed germination and seedling development in Arabidopsis. Plant Physiol. 163:318-330.

Steinkellner, S., Lendzemo, V., Langer, I., Schweiger, P., Khaosaad, T., Toussaint, J.-P., and Vierheilig, H. 2007. Flavonoids and strigolactones in root exudates as signals in symbiotic and pathogenic plant-fungus interactions. Molecules 12:1290-1306.

Stes, E., Depuydt, S., De Keyser, A., Matthys, C., Audenaert, K., Yoneyama, K., Werbrouck, S., Goormachtig, S., and Vereecke, D. 2015. Strigolactones as an auxiliary hormonal defence mechanism against leafy gall syndrome in Arabidopsis thaliana. J. Exp. Bot. 66:5123-5134.

Stirnberg, P., Furner, I. J., and Leyser, H. M. O. 2007. MAX2 participates in an SCF complex which acts locally at the node to suppress shoot branching. Plant J. 50:80-94.

Tambalo, D. D., Vanderlinde, E. M., Robinson, S., Halmillawewa, A., Hynes, M. F., and Yost, C. K. 2014. Legume seed exudates and Physcomitrella patens extracts influence swarming behavior in Rhizobium leguminosarum. Can. J. Microbiol. 60:15-24.

Toh, S., Holbrook-Smith, D., Stogios, P. J., Onopriyenko, O., Lumba, S., Tsuchiya, Y., Savchenko, A., and McCourt, P. 2015. Structure-function analysis identifies highly sensitive strigolactone receptors in Striga. Science 350:203-207.
Tokunaga, T., Hayashi, H., and Akiyama, K. 2015. Medicaol, a strigolactone identified as a putative didehydro-orobanchol isomer, from Medicago truncatula. Phytochemistry 111:91-97.

Torres-Vera, R., García, J. M., Pozo, M. J., and López-Ráez, J. A. 2014. Do strigolactones contribute to plant defence? Mol. Plant Pathol. 15:211-216.

Tsuchiya, Y., and McCourt, P. 2009. Strigolactones: A new hormone with a past. Curr. Opin. Plant Biol. 12:556-561.

Umehara, M., Hanada, A., Yoshida, S., Akiyama, K., Arite, T., TakedaKamiya, N., Magome, H., Kamiya, Y., Shirasu, K., Yoneyama, K., Kyozuka, J., and Yamaguchi, S. 2008. Inhibition of shoot branching by new terpenoid plant hormones. Nature 455:195-200.

Venturi, V., and Keel, C. 2016. Signaling in the rhizosphere. Trends Plant Sci. 21:187-198.

Vurro, M., Prandi, C., and Baroccio, F. 2016. Strigolactones: How far is their commercial use for agricultural purposes? Pest Manag. Sci. 72:2026-2034.

Wang, L., Wang, B., Jiang, L., Liu, X., Li, X., Lu, Z., Meng, X., Wang, Y., Smith, S. M., and Li, J. 2015. Strigolactone signaling in Arabidopsis regulates shoot development by targeting D53-like SMXL repressor proteins for ubiquitination and degradation. Plant Cell 27:3128-3142.

Waters, M. T. 2017. From little things big things grow: Karrikins and new directions in plant development. Funct. Plant Biol. 44:373-385.

Waters, M. T., Gutjahr, C., Bennett, T., and Nelson, D. C. 2017. Strigolactone signaling and evolution. Annu. Rev. Plant Biol. 68:291-322.

Waters, M. T., Nelson, D. C., Scaffidi, A., Flematti, G. R., Sun, Y. K., Dixon, K. W., and Smith, S. M. 2012. Specialisation within the DWARF14 protein family confers distinct responses to karrikins and strigolactones in Arabidopsis. Development 139:1285-1295.

Wei, X., and Bauer, W. D. 1999. Tn5-induced and spontaneous switching of Sinorhizobium meliloti to faster-swarming behavior. Appl. Environ. Microbiol. 65:1228-1235.

Xie, X. 2016. Structural diversity of strigolactones and their distribution in the plant kingdom. J. Pestic. Sci. 41:175-180.

Xie, X., Wang, G., Yang, L., Cheng, T., Gao, J., Wu, Y., and Xia, Q. 2015a. Cloning and characterization of a novel Nicotiana tabacum ABC transporter involved in shoot branching. Physiol. Plant. 153:299-306.

Xie, X., Yoneyama, K., Kisugi, T., Nomura, T., Akiyama, K., Asami, T., and Yoneyama, K. 2015b. Strigolactones are transported from roots to shoots, although not through the xylem. J. Pestic. Sci. 40:214-216.

Xu, Y., Miyakawa, T., Nakamura, H., Nakamura, A., Imamura, Y., Asami, T., and Tanokura, M. 2016. Structural basis of unique ligand specificity of KAI2-like protein from parasitic weed Striga hermonthica. Sci. Rep. 6:31386.

Yoneyama, K., Arakawa, R., Ishimoto, K., Kim, H. I., Kisugi, T., Xie, X., Nomura, T., Kanampiu, F., Yokota, T., Ezawa, T., and Yoneyama, K. 2015. Difference in Striga-susceptibility is reflected in strigolactone secretion profile, but not in compatibility and host preference in arbuscular mycorrhizal symbiosis in two maize cultivars. New Phytol. 206:983-989.

Yoneyama, K., Awad, A. A., Xie, X., Yoneyama, K., and Takeuchi, Y. 2010. Strigolactones as germination stimulants for root parasitic plants. Plant Cell Physiol. 51:1095-1103.

Yoneyama, K., Xie, X., Kisugi, T., Nomura, T., and Yoneyama, K. 2013. Nitrogen and phosphorus fertilization negatively affects strigolactone production and exudation in sorghum. Planta 238:885-894.

Zhang, Y., van Dijk, A. D. J., Scaffidi, A., Flematti, G. R., Hofmann, M., Charnikhova, T., Verstappen, F., Hepworth, J., van der Krol, S., Leyser, O., Smith, S. M., Zwanenburg, B., Al-Babili, S., Ruyter-Spira, C., and Bouwmeester, H. J. 2014. Rice cytochrome P450 MAX1 homologs catalyze distinct steps in strigolactone biosynthesis. Nat. Chem. Biol. 10: 1028-1033.

Zhao, L.-H., Zhou, X. E., Yi, W., Wu, Z., Liu, Y., Kang, Y., Hou, L., de Waal, P. W., Li, S., Jiang, Y., Scaffidi, A., Flematti, G. R., Smith, S. M., Lam, V. Q., Griffin, P. R., Wang, Y., Li, J., Melcher, K., and Xu, H. E. 2015. Destabilization of strigolactone receptor DWARF14 by binding of ligand and E3-ligase signaling effector DWARF3. Cell Res. 25:1219-1236.

Zheng, K., Wang, X., Weighill, D. A., Guo, H.-B., Xie, M., Yang, Y., Yang, J., Wang, S., Jacobson, D. A., Guo, H., Muchero, W., Tuskan, G. A., and Chen, J.-G. 2016. Characterization of DWARF14 genes in Populus. Sci. Rep. 6:21593.

Zhou, F., Lin, Q., Zhu, L., Ren, Y., Zhou, K., Shabek, N., Wu, F., Mao, H., Dong, W., Gan, L., Ma, W., Gao, H., Chen, J., Yang, C., Wang, D., Tan, J., Zhang, X., Guo, X., Wang, J., Jiang, L., Liu, X., Chen, W., Chu, J., Yan, C., Ueno, K., Ito, S., Asami, T., Cheng, Z., Wang, J., Lei, C., Zhai, H., Wu, C., Wang, H., Zheng, N., and Wan, J. 2013. D14-SCF ${ }^{\mathrm{D} 3}$-dependent degradation of D53 regulates strigolactone signalling. Nature 504:406-410.

Zwanenburg, B., and Pospíšil, T. 2013. Structure and activity of strigolactones: New plant hormones with a rich future. Mol. Plant 6:38-62. 